\title{
Design Methods for 3D RFID Antennas Located on a Conducting Ground Plane
}

\author{
Amir Galehdar, David V. Thiel, Senior Member, IEEE, and Steven G. O'Keefe, Member, IEEE
}

\begin{abstract}
Based on 2D meander line methods, $3 \times 3 \times 3$ point, symmetrical, 3D dipole antennas structure was investigated exhaustively to identify those configurations with the highest efficiency and lowest resonant frequency for a fixed length. Maximum efficiency and input impedance occurs when adjacent high current segments in the antenna are oppositely directed. The optimal antenna structure was compressed and the effect of a nearby conducting plane was investigated. The meander line structure greatly improves antenna performance with a resonant frequency of $498 \mathrm{MHz}$ and an efficiency of $62 \%$ when located on a conducting ground plane.
\end{abstract}

Index Terms-Antenna efficiency, Hilbert curve, Numerical Electromagnetic Code (NEC), Radio Frequency Identification (RFID), wire antenna.

\section{INTRODUCTION}

$\mathbf{R}$ ADIO frequency identification (RFID) is an emerging technology for remotely identifying assets and controlling devices via a wireless link. A typical RFID transponder (tag) consists of an antenna and an integrated circuit chip which stores an identification number and other information. Tags can be classified as [1]:

- fully-active where the battery powers the microcontroller and radio transmitter;

- semi-active when the local source feeds the sensors;

- passive (no battery) where the querying transmitted signal from the reader consists of modulated information with periods of unmodulated carrier which is converted to electrical power to run the circuit.

The passive RFID tags are usually the least expensive and most reliable and so are popular for tracking and managing assets. The biggest problem with traditional tags is the effect of metal surfaces and other nearby objects on their performance. This can have a devastating effect on efficiency of the antenna. Not only does this influence the reading range in passive tags but also affects the operating life expectancy of active tags. The efficiency of an antenna while influenced by many factors, is affected by structure as studied in this research.

Manuscript received January 24, 2008; revised September 04, 2008. Current version published March 20, 2009.

The authors are with the Centre for Wireless Monitoring and Applications, Griffith University, Nathan Qld 4111, Australia (e-mail: Amir.MohammadzadehGalehdar@student.griffith.edu.au; d.thiel@griffith.edu.au; s.okeefe@ griffith. edu.au).

Color versions of one or more of the figures in this paper are available online at http://ieeexplore.ieee.org.

Digital Object Identifier 10.1109/TAP.2008.2011227
Typical UHF tags use silver ink, printed dipole antennas. The most popular planar miniature UHF antennas for RFID tags are spirals [2]-[5], loops [5]-[7] and meander lines [8]-[13]. The common design objective is to obtain the best performance in a fixed surface area [14].

Galehdar et al. [13] reported a study of planar RFID meander antennas created by connecting tracks through a rectangular array of points. In this way, all possible meander structures in the area were found. By exhaustively simulating all possible structures, both the most efficient and the biggest electrical size antenna in the family were found. This study covered all meander shapes including the Hilbert curve antenna. Some researchers considered planar (two dimensional) structures based on the Hilbert curve [15]-[17] and extended the idea to a cube where three dimensional Hilbert antenna was also discussed [18].

In this paper the results from a comprehensive study of three dimensional meander line antennas are presented, and a new design method is introduced. All antennas were simulated using NEC [19] and their efficiency and input impedance at the resonant frequency were determined. All antennas in a given cube with fixed length and segment size were compared and the results for all possible meander structures are presented. The most efficient antenna was chosen and its capacity to function reliably while located on a conducting surface was investigated. Once determined, the height was reduced so that the structure is almost two dimensional.

\section{MethOD}

For a given volume, an infinite number of wire antennas can be designed. Altshuler [20] used an optimization method to find the best performing antenna within a size limitation. This paper extends this concept and presents a new method of creating designs based on a logical design perspective.

A regular, rectangular grid following [13] was introduced in three dimensions. The only difference is that a volume or cube was mapped which constrains each half of a symmetrical antenna to a fixed length, volume and segment size. In order to investigate a finite number of antenna designs, a set of 27 $(3 \times 3 \times 3)$ grid points in the cube were chosen. The aim was to develop a method for finding the best performing antenna via an exhaustive study exploring all possible configurations. This is a time consuming procedure. In future work, this exhaustive study may not be required as optimization of this type meander antennas using the ant colony method shows promise [21]. Optimization can reduce the number of structures simulated and so reduce the time required to find the best structure. 


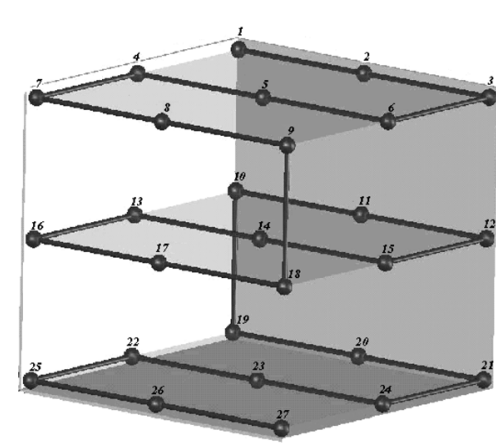

(a)

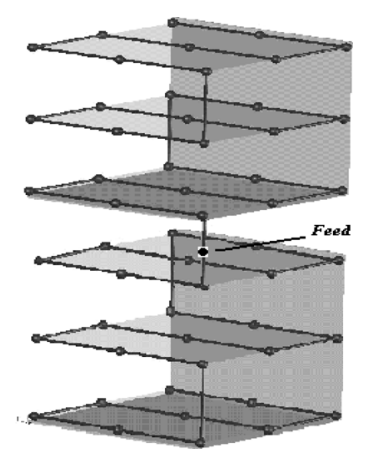

(b)
Fig. 1. (a) An example of a cubic meander line. (b) The complete antenna with feed point.

A $2 \times 2 \times 2 \mathrm{~cm}^{3}$ cube was chosen to produce one half of a center fed, symmetrical dipole mapped by using all 27 grid points. The points are $1 \mathrm{~cm}$ apart and, when all are connected, a $26 \mathrm{~cm}$ long wire is produced. This is illustrated in Fig. 1(a). The dipole was completed using a mirror image of the design and a $1 \mathrm{~cm}$ long connection between the two cubes. The feed was located in the center of this connection. This means the total length of the antenna is $53 \mathrm{~cm}$ [see Fig. 1(b)] and the total volume of the dipole is $2 \times 2 \times 5 \mathrm{~cm}^{3}$.

In order to find all possible combinations, the 27 grid points in the half volume were numbered and the following connection rules applied.

- The meander line starts from one point on the line of symmetry to allow connection to the other half of the dipole (a symmetrical cubic cell);

- The meander line passes through each grid point once only;

- Only 90 degree corners are permitted. (For example, this means 1 can be connected to 2, 4 or 10 only);

- Only three feed points on the line of symmetry were considered because the other points produce repeated patterns. Thus only points 1,2 and 5 are unique starting points.

All possible permutations joining these 27 points that generate a full length meander line antenna were produced by following these rules and applying the tree algorithm [22]. Based on the algorithm, a start point was selected (for example grid point 1 ), then the algorithm finds the next possible points and expands the starting point into a array of all possible combinations (e.g. $\left.\left\{\begin{array}{ll}1 & 2\end{array}\right\},\left\{\begin{array}{ll}1 & 4\end{array}\right\},\left\{\begin{array}{ll}1 & 10\end{array}\right\}\right)$. The last member of each array was chosen as a start point and all possible combinations expand. If the line gets to a point where no expansion was possible to include all 27 points, that line was terminated and rejected. Based on this method, 392628 possible permutations were found using start point 1. Start point 5 generates 303262 possible permutations. Start point 2 does not produce any permutations. Thus the total number of permutations was 695890. These strings of numbers were translated into coordinates in space and a mirror image produced to complete the dipole. These coordinates were translated into a NEC input file using a wire radius of $0.05 \mathrm{~mm}$ and a wire conductivity of $5.8 \times 10^{7} \mathrm{~S} / \mathrm{m}$. All structures were simulated across the frequency band from 300 to $500 \mathrm{MHz}$ with a step of $5 \mathrm{MHz}$. The aim is to design an RFID antenna for $433 \mathrm{MHz}$ band [23], [24]

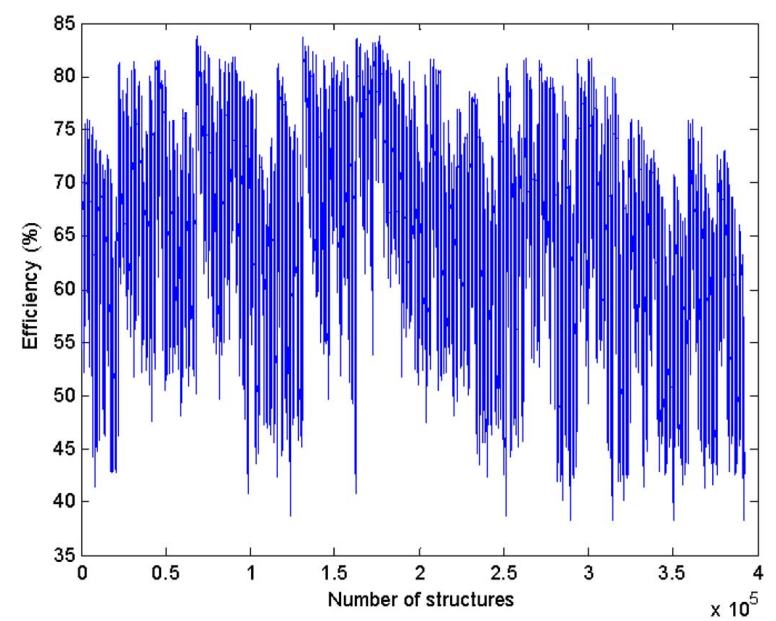

Fig. 2. Efficiency values for all structures using feed 1.

but since there were more than 700000 different structures some of the results have gone off the ideal band although they all have the same physical length. The uncompromised results were presented so they can be comparable. The input impedance, resonant frequency and current distribution at the resonant frequency of all the structures were calculated.

The antenna efficiency was calculated using the method introduced in [25]. The wire structure is divided into small segments and the power loss over each segment was calculated, then the efficiency $\eta$ was calculated using

$$
\eta=\frac{R_{\mathrm{in}} I^{2}-\sqrt{\frac{\pi f \mu_{0}}{\sigma}} \sum_{i=1}^{N} \frac{l_{i} i_{i}^{2}}{2 \pi a_{i}}}{R_{\mathrm{in}} I^{2}} .
$$

where $R_{\text {in }}$ is the input resistance (radiation plus loss resistance), $I$ is the current of the modeled RLC circuit, $R_{s}$ is surface resistance of wire, $f$ is the frequency of operation, $\mu_{0}$ is the permittivity of free space, $\sigma$ is the conductivity of the wire, $a_{i}$ and $l_{i}$ are the diameter and length of the $i$ th segment respectively.

\section{RESULTS}

There are well known limitations to the performance characteristics of electrically small antennas [26]. Antenna performance can be improved within these limits. Usually antenna engineers seek to define structures with the smallest resonant frequency and highest efficiency for a given area or volume. In this case, the results are presented in terms of resonant frequency and efficiency of all possible structures. Data is presented separately for the two different feed points. The input impedances were calculated, but since these structures are electrically small, the impedances are relatively small so that matching circuits are mandatory for a practical implementation of the antenna.

\section{A. Efficiency}

The efficiency of all the structures have been calculated at their resonant frequencies and results are presented in two different categories based on the feed positions. The efficiency of the structures using feed 1 and feed 5 are plotted in Figs. 2 and 3 , respectively. 
TABLE I

SUMMARIZED RESULTS OF EFFICIENCY FOR BOTH FEED POINTS

\begin{tabular}{|c|c|c|c|c|c|}
\cline { 3 - 6 } \multicolumn{2}{c|}{} & Efficiency (\%) & Res. Freq. (Mhz) & Input Impedance (Ohm) & No. of Struc. \\
\hline \multirow{2}{*}{ feed1 } & $\max 1$ & 83.7 & 475 & 3.4 & 68557 \\
\cline { 2 - 6 } & $\min 1$ & 38.2 & 365 & 1 & 350934 \\
\hline \multirow{2}{*}{ feed5 } & $\max 5$ & 83.3 & 465 & 3.2 & 847 \\
\cline { 2 - 6 } & $\min 5$ & 32.1 & 345 & 0.9 & 294773 \\
\hline
\end{tabular}

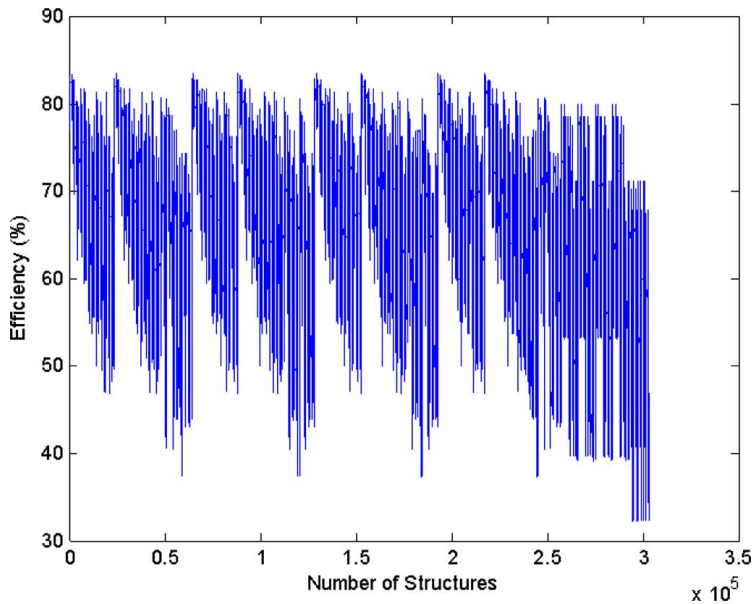

Fig. 3. Efficiency values for all structures using feed 5.

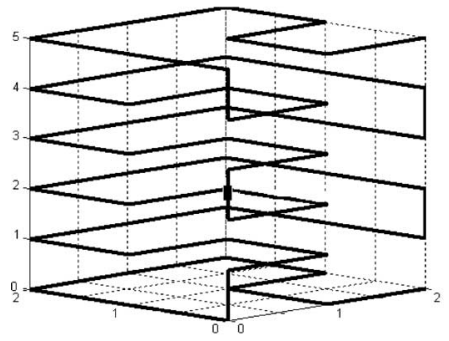

(a)

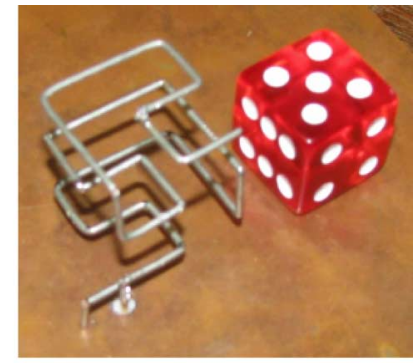

(b)
Fig. 4. (a) The most efficient structure (all sizes in $\mathrm{cm}$ ), (b) the monopole structure on the ground plane (standard dice).

Over all the feed point 1 has more structures with efficiency higher than $80 \%$ and alternatively the feed point 5 contains the structure with the lowest efficiency. This means high efficient structures are the ones with feed point is on their side.

There are two structures with the smallest efficiency of $38.2 \%$ and two structures have the biggest efficiency of $83.7 \%$. These results are summarized in Table I.

In order to confirm these results, the most efficient structure [see Fig. 4(a)] was built and S11 is measured practically. Since this dipole antenna is relatively small and connecting a SMA connector to feed it at $475 \mathrm{MHz}$ with Bazooka Balun (which will be huge compare to antenna) is a difficult task, a monopole structure has been made and simulated. ${ }^{1}$

A $12 \times 12 \mathrm{~cm}^{2}$ Copper sheet is used as a ground plane and since the input impedance of the antenna is very low for VNA offset feeding is used to increase the input impedance [see Fig. 4(b)] to match to 50 ohms. A low $1 \mathrm{~m}$ semi rigid cable

\footnotetext{
${ }^{1}$ The most common standard dice is $1.87 \mathrm{~cm}(3 / 4 \mathrm{inch})$ on each side.
}

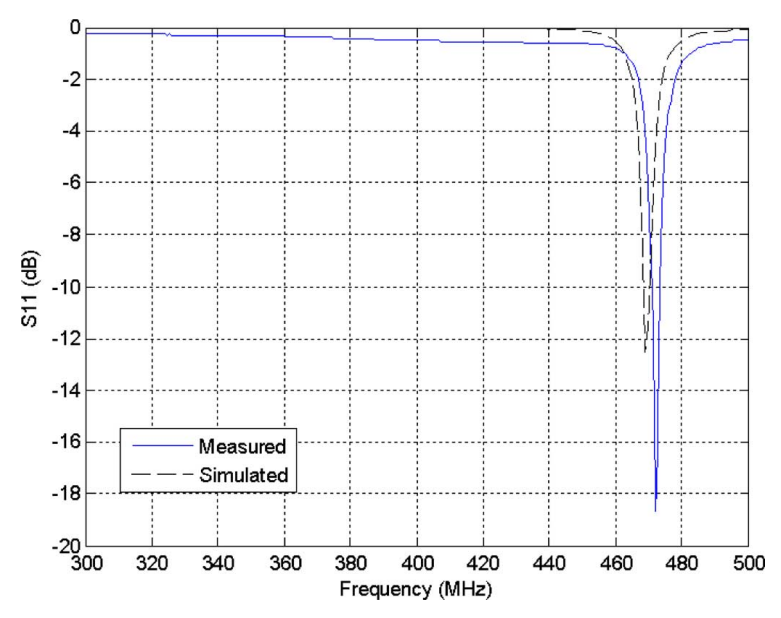

Fig. 5. S11 simulation and practical results of the most efficient monopole structure.

with mounted ferrites bead is used in practical measurements to reduce the surface current and increase accuracy.

The antenna is $0.5 \mathrm{~cm}$ above the ground plane and the coupled size needs to be $0.5 \mathrm{~cm}$ away from antenna and $0.5 \mathrm{~cm}$ above the ground plane. These sizes were found by trial and error not by optimization so better matching is possible. The feeding point is offset by $1.77 \mathrm{~cm}$ from the center of the ground plane so the center point of the structure is aligned with the center of the ground.

A good agreement can be seen in Fig. 5 between the simulation and measured results. The simulation results are calculated in NEC where the ground plane is built with mesh of Copper wires with $0.2 \mathrm{~cm}$ distance from each other.

\section{B. Resonant Frequency}

The resonant frequency in this research is defined as the frequency where the imaginary part of input impedance is zero. The resonant frequency with real input impedance has been chosen as a reference point for all the structures so any other point on the frequency response can be chosen for comparison. The resonant frequency is plotted in Fig. 6 for all possible feed 1 structures. The "structure number" on the X-axis is an arbitrary reference number for each structure.

The smallest resonant frequency was $350 \mathrm{MHz}$ and there were two structures with that resonant frequency. The highest resonant frequency in this category was $475 \mathrm{MHz}$ and 91 structures resonate at that frequency. The results for feed 5 are given in Fig. 7 where the frequencies are distributed from $345-470 \mathrm{MHz}$. There are 8 structures resonant at the lowest and 80 structures at the highest value in this category. The discontinuity in Fig. 6 and 
TABLE II

SuMMARY OF THE FREQUENCY RESULTS FOR BOTH FEEDS

\begin{tabular}{|c|c|c|c|c|c|}
\cline { 3 - 6 } \multicolumn{2}{c|}{} & Res. Freq. (Mhz) & Efficiency (\%) & Input Impedance (Ohm) & No. of struc. \\
\hline \multirow{2}{*}{ feed1 } & $\max 1$ & 475 & 83.6 & 3.4 & 68553 \\
\cline { 2 - 6 } & $\min 1$ & 350 & 43.5 & 1.1 & 281636 \\
\hline \multirow{2}{*}{ feed5 } & $\max 5$ & 470 & 82.9 & 3.2 & 864 \\
\cline { 2 - 6 } & $\min 5$ & 345 & 32.1 & 0.9 & 294773 \\
\hline
\end{tabular}

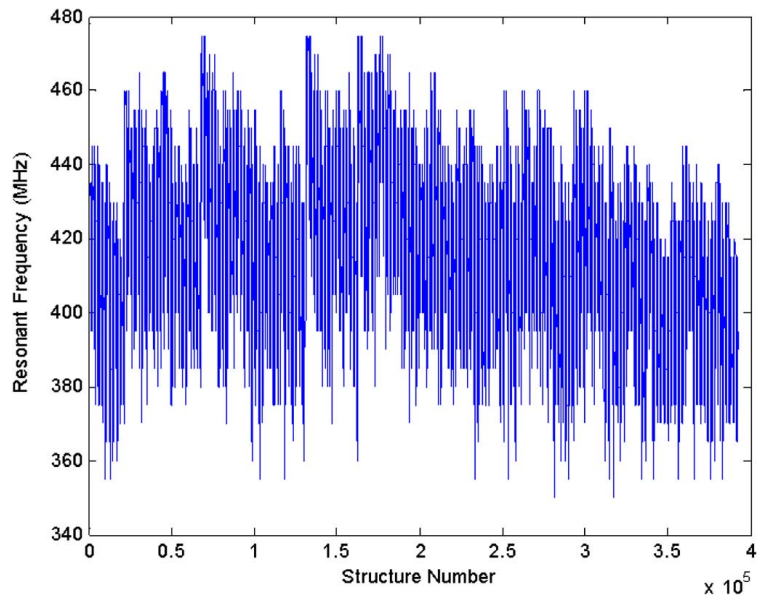

Fig. 6. The resonant frequency for all structures using feed 1 .

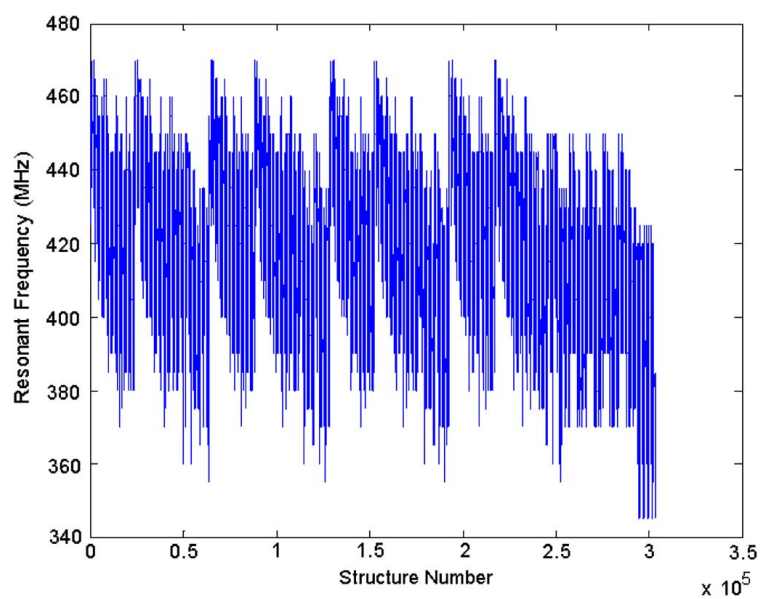

Fig. 7. The resonance frequency for all structures using feed 5 .

7 is caused by the $5 \mathrm{MHz}$ steps in the frequency sweep which means the resolution is $5 \mathrm{MHz}$.

These results are summarized in Table II where the lowest frequency in both categories was $345 \mathrm{MHz}$. The structure with lowest resonant frequency which can be seen in Fig. 8, was chosen to be built and the S11 and resonant frequency are measured practically.

The same set up and ground plane as before is used to do the test, but the coupling point is found to be $0.4 \mathrm{~cm}$ above the ground and $0.5 \mathrm{~cm}$ away from the antenna. The antenna is mounted $0.5 \mathrm{~cm}$ above the ground plane since the feed in dipole was $1 \mathrm{~cm}$. same structure is simulated in NEC with same mesh ground and results can be seen in Fig. 9.

A good agreement can be observed in Fig. 9 between the practical and measured results. It can be seen that the bandwidth is

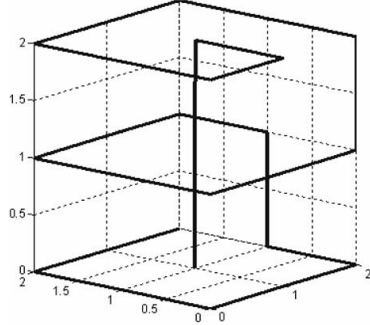

(a)

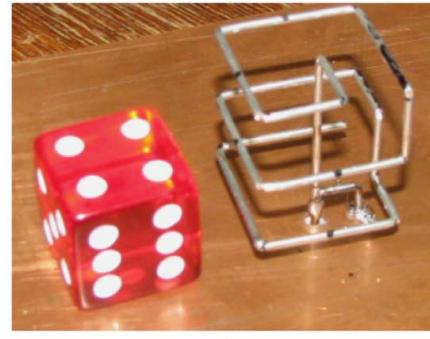

(b)
Fig. 8. (a) It is the largest electrical size antenna (all sizes in centimeters). (b) The monopole structure on the ground plane (standard dice).

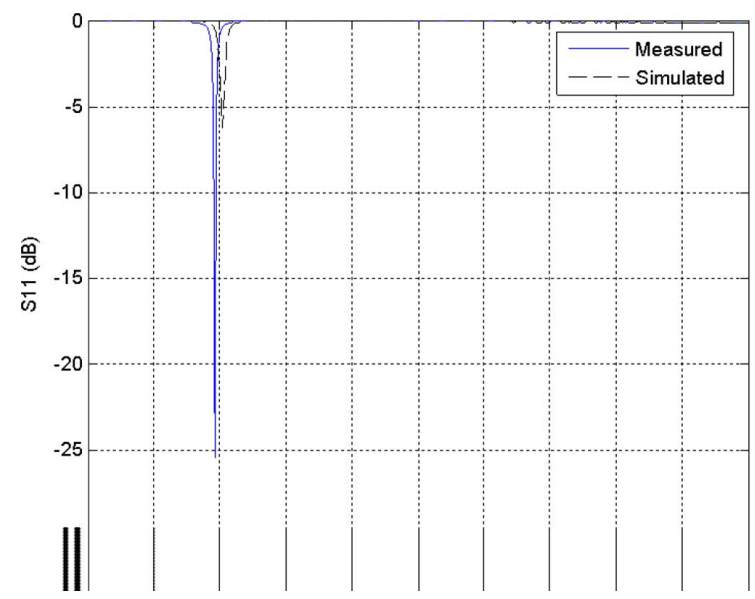

Fig. 9. The largest electrical size antenna results simulation and practical.

narrower compared to Fig. 5 since the coupling is higher and that is reason for the low frequency.

\section{Multiobjective Optimization}

The highest efficiency and the lowest resonant frequency are not found in the same structure so a compromise is required. For example in Tables I and II, the structure with the lowest resonant frequency also has the smallest efficiency. In Fig. 10, the efficiency and resonant frequency is plotted for all possible antennas. It can be seen that the highest efficiency usually contains the maximum resonant frequency.

\section{DISCUSSION}

\section{A. Efficiency}

It is useful for designers to develop an understanding of the structural effects on the antenna properties. The structure of a common meander line dipole is shown in Fig. 11, together with the radiation pattern. The radiation pattern is similar to that for a 


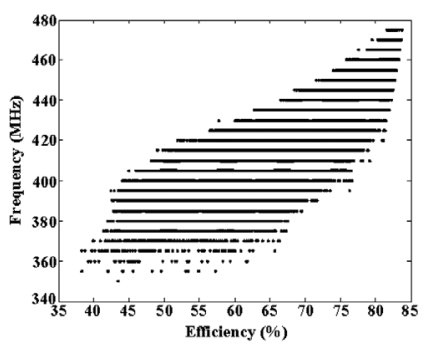

(a)

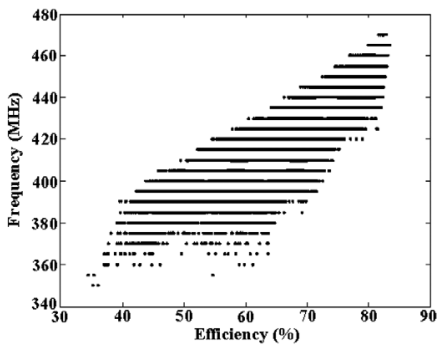

(b)
Fig. 10. (a) Scatter plot showing the efficiency verses frequency for feed 1 and (b) feed 5 .
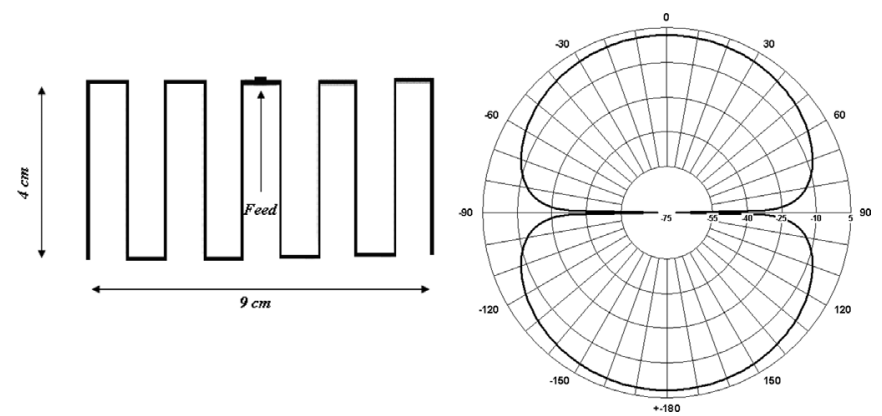

Fig. 11. Structure and radiation pattern (in decibels) of a common meander line antenna.

horizontal straight dipole which suggests that the horizontal segments mainly contribute to the radiation while the vertical segments increase the effective length and provide inductive load which reduces the resonant frequency. This research suggests that all segments play a role in the efficiency. The folded segments with high current lies in opposite directions and the radiation resistance increases because the current flow is limited. This lowers the loss and increases efficiency.

The opposite effect is evident in the structures shown in Fig. 13(b), where high current segments are not positioned with current in opposite directions. This lowers the input resistance and lowers the efficiency. The same principles apply to 3D structures. In this case the vertical segments contribute to the radiation and all other segments contribute to the inductive load which lowers the resonant frequency. If the folds are positioned in high current areas and close to other segments with current in opposite directions, the structure has high efficiency. One half of the most efficient structure in both families is shown in Fig. 12. The segments close to the feed (high current areas) are positioned next to each other with oppositely directed current. This reduces the current magnitude which means lower loss and higher efficiency.

This theory is confirmed in Table I where the least efficient structures have low input impedance. The structure with the lowest efficiency and input impedance is also shown in Fig. 13(a).

One can conclude that the most efficient structures are those with folding in high current areas with adjacent segments having oppositely directed current.

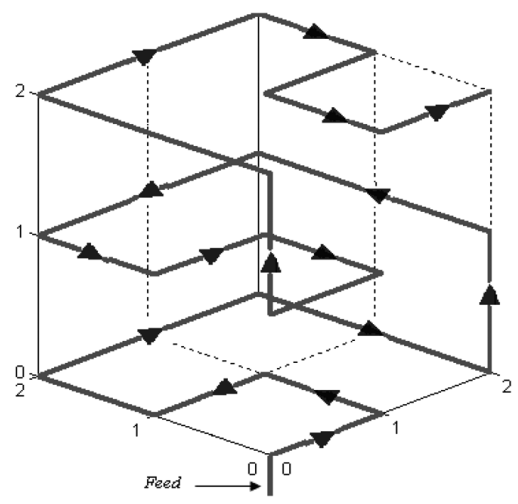

Fig. 12. The structure with highest efficiency (all the sizes are in centimeters).

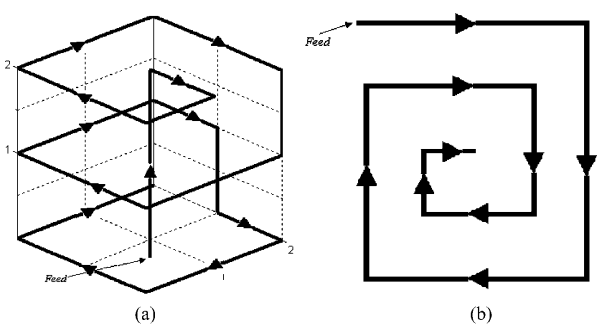

Fig. 13. Current direction in the (a) 3D structure with the lowest resonant frequency and (b) 2D structure with the lowest resonant frequency.

\section{B. Frequency}

The half the structure with the lowest resonant frequency is plotted in Fig. 13(a). There are similarities between this structure and the structure with the lowest resonant frequency in $2 \mathrm{D}$ (Fig. 13(b) [13]). As the antennas are electrically small, the current in each segment is in phase. Fig. 13 shows the direction of the current in both the 2D and 3D structures at resonance. The current magnitude decreases along the wire and so those segments closest to the feed point carry the highest currents.

The current segments in the best structures are arranged such that high current segments are well separated and adjacent (nearest) segments have currents in same direction.

In these structures the magnetic flux is maximized because all segments have current in the same circulating direction. This means the inductive load is higher and so the resonant frequency is lower. These structures are not necessarily the best structures in terms of resonant frequencies in their given areas or volume, because by simply gridding the same area with more points structures with smaller resonant frequencies could be found. Based on the design rules, this structure has the lowest frequency in the family.

\section{RFID DESIGN}

In Sections II and III, the design principle and the best 3D structures were shown. These 3D antennas are clearly much thicker than 2D antennas which for some applications is considered a disadvantage. In this Section, the advantage of the 3D antennas over $2 \mathrm{D}$ is presented in the form of a sample case study.

It is well known that planar RFID antennas do not operation effectively on conductive surfaces although many methods have been suggested to resolve this problem [27]-[29] in here a smaller alternative option is suggested. 
TABLE III

3D ANTENNA PERFormance BeFore AND AFTER Z COMPRESSION.

\begin{tabular}{|c|c|c|c|c|}
\cline { 2 - 5 } \multicolumn{1}{c|}{} & Efficiency (\%) & Res. Freq. (Mhz) & Input Impedance (Ohm) & Gain (dBi) \\
\hline Before com. & 83.7 & 475 & 3.4 & 1.242 \\
\hline After com. & 54.3 & 598 & 1.8 & -6.51 \\
\hline
\end{tabular}

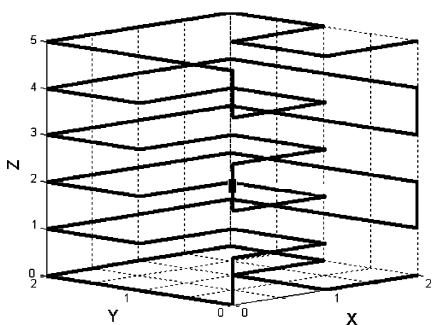

(a)

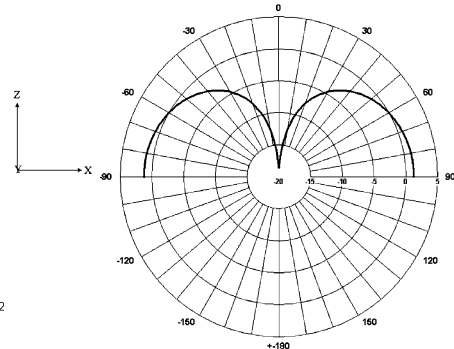

(b)
Fig. 14. (a) The most efficient 3D dipole structure. (b) Radiation pattern in decibels.

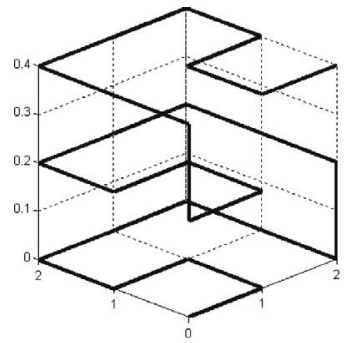

(a)

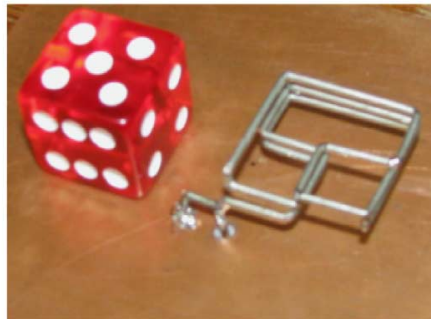

(b)
Fig. 15. (a) The most efficient 3D dipole structure compressed (sizes in centimeters). (b) Monopole antenna (standard dice).

The strategy is to reduce the vertical dimension of the 3D antennas, while leaving the other two dimensions unchanged. This modified structure was investigated when located above a perfect ground plane. The most efficient 3D structure [see Fig. 4(a)] was used as the basis for comparison.

\section{A. Compact 3D Dipole: Free Space}

The complete dipole structure shown in Fig. 14(a) fits into a volume $5 \times 2 \times 2 \mathrm{~cm}^{3}$. The radiation pattern is shown in Fig. 14(b). The principal direction of the radiation is perpendicular to the dipole as expected. The segments oriented in the $\mathrm{Z}$ direction dominate the radiation pattern, whereas the segments oriented in the $\mathrm{X}$ and $\mathrm{Y}$ axes contribute to the inductive load and reduce the resonant frequency. Logic suggests that the compression needs to be in $\mathrm{Z}$ axis. This will move the non radiating elements closer together, and the effect on the antenna performance will be minimal. So, the $\mathrm{Z}$ elements have been reduced to $1 / 5$ th of their values which causes the overall height of antenna to be $1 \mathrm{~cm}$.

This means each of the $\mathrm{Z}$ segments of antenna has $0.2 \mathrm{~cm}$ length (instead of $1 \mathrm{~cm}$ ). This is illustrated in Fig. 15 and the change in antenna performance is summarized in Table III.

The resonant frequency increased, partly because the total wire length reduced from $53 \mathrm{~cm}$ to $49 \mathrm{~cm}$. While the volume was reduced by $7.5 \%$, the resonant frequency was increased by $21 \%$. This can be partly explained in terms of the increase in capacitance between the $\mathrm{X}$ and $\mathrm{Y}$ directed elements in different

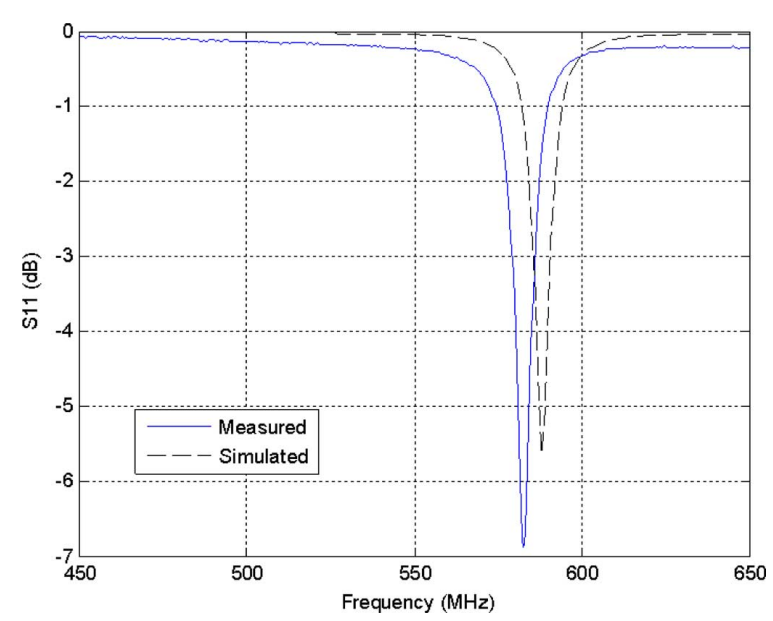

Fig. 16. The squashed structure test.

layers. Clearly the inductance also increased but the capacitance change was more significant.

The efficiency reduced to $54 \%$. The compression resulted in an overall $\mathrm{Z}$ length reduction of $80 \%$ (from 5 to $1 \mathrm{~cm}$ ), but there is only a $30 \%$ loss in efficiency. This is thought to be because opposing high current segments are now much closer together. The gain was reduced to almost one sixth of the original value. This is close to the $80 \%$ reduction of the $\mathrm{Z}$ length of the radiation elements.

In order to confirm these results the antenna has been made and tested. Once again due to the same limitation as mentioned before, a monopole was built and a coupled feed is used. The coupled feed size was $0.4 \mathrm{~cm}$ high and $0.5 \mathrm{~cm}$ long. Thus, the feed to the structure is mounted $0.5 \mathrm{~cm}$ above the ground which is $0.4 \mathrm{~cm}$ higher than the dipole compressed design.

A good agreement can be observed in Fig. 16 between practical and measured reflection coefficient. However, the resonant frequency is displaced compared to dipole compressed structure; this can be due to increasing the height to a ground plane in monopole testing.

\section{B. Compact 3D Dipole: Ground Plane}

The antenna shown in Fig. 13(a) was positioned $0.2 \mathrm{~cm}$ above a perfect ground plane (see Fig. 17). The distance above the ground plane was chosen to be very small compared to one free space wavelength $\lambda_{0}=54 \mathrm{~cm}$. The null point in the radiation pattern was found to be less intense. This is considered a useful improvement as usually the RFID reader is placed inline and parallel to the antenna. The efficiency, gain and input impedance of the antenna increased when compared to the same antenna in free space (see Table IV).

Based on these observations, not only does the ground not significantly affect antenna performance, but some antenna parameters have been improved. The resonant frequency decreased, 
TABLE IV

ANTENNA PERformance With Ground Plane

\begin{tabular}{|c|c|c|c|c|}
\cline { 2 - 5 } \multicolumn{1}{c|}{} & Efficiency (\%) & Res. Freq. (Mhz) & Input Impedance (Ohm) & Gain (dBi) \\
\hline Com. With Ground & 63.5 & 558 & 2.1 & -1.84 \\
\hline
\end{tabular}

TABLE V

3D Diagonal Antenna Performance With Ground Plane

\begin{tabular}{|c|c|c|c|c|}
\cline { 2 - 5 } \multicolumn{1}{c|}{} & Efficiency (\%) & Reso. Freq. (Mhz) & Input Impedance (Ohm) & Gain (dBi) \\
\hline Before com. & 80.5 & 470 & 3.3 & 1.1 \\
\hline After com. & 55.2 & 535 & 1.8 & -8.3 \\
\hline Com. With Ground & 61.8 & 498 & 2 & -3.3 \\
\hline
\end{tabular}

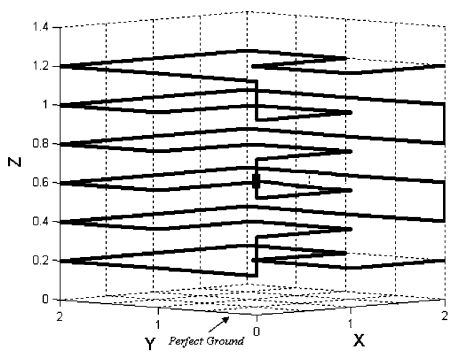

(a)

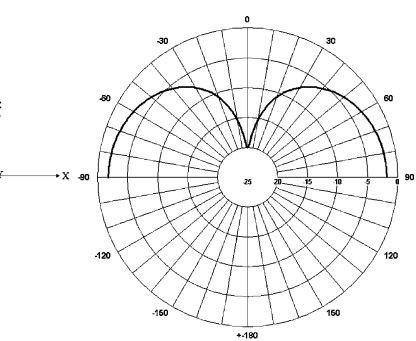

(b)

Fig. 17. (a) 3D antenna with ground plane. (b) Radiation pattern of the antenna in decibels.

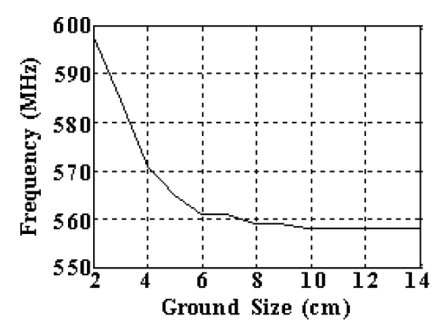

(a)

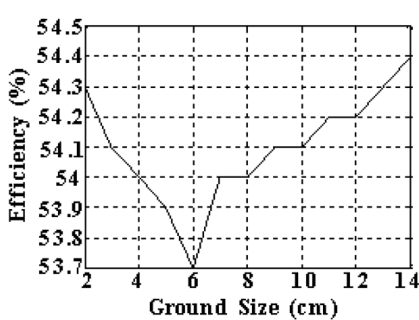

(b)

Fig. 18. (a) Resonant frequency effected by ground size. (b) Efficiency effected by ground size.

the gain has almost doubled ( $6 \mathrm{~dB}$ increase) and the efficiency improved by $10 \%$. The one slight disadvantage is that there is still a radiation null in $\mathrm{Z}$ direction. This means, if this antenna is used as an RFID tag, the reader is best placed at a 30 degree angle from the tag. This may be a positive point because, in the near field, the metal surface may affect the reader antenna also.

The effects of different ground sizes on squashed dipole antenna have been investigated. This has been done by simulating different ground sizes near the antenna and the resonant frequency and efficiency are measured for each case (see Fig. 18).

The ground is simulated in NEC by producing a perfect (no loss) mesh of wires placed $0.5 \mathrm{~cm}$ from each other. It can be seen that as the ground size increases from $2 \times 2$ to $10 \times 10 \mathrm{~cm}^{2}$ the resonant frequency reduces from 598 to $558 \mathrm{MHz}$. Additionally, it can be concluded that for the grounds bigger than $10 \times 10 \mathrm{~cm}^{2}$, the effect is as same as a $10 \times 10 \mathrm{~cm}^{2}$ ground. This is confirmed with the results of infinite ground plane where the resonant frequency has been measured to be $558 \mathrm{MHz}$ (see Table IV). This means if the tag is matched for $10 \times 10 \mathrm{~cm}^{2}$ ground then it can be placed on any conductive surface as long as it is as big as or bigger than $10 \times 10 \mathrm{~cm}^{2}$.

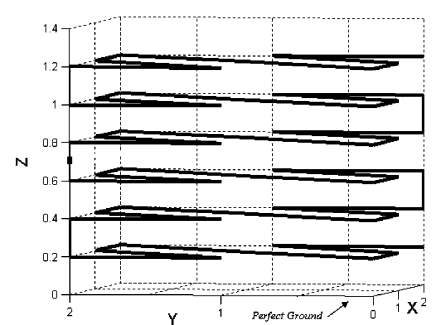

(a)

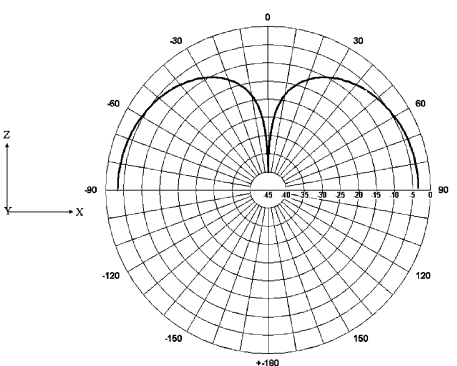

(b)
Fig. 19. (a) 3D squashed antenna using diagonals with ground plane. (b) Radiation pattern.

Further improvements were observed using a slight design modification. The investigation was not exhaustive as only one such design was introduced. Although this structure is not a member of this family defined in Section II, it was constructed by the same connecting points and allowing 45 degree corners. The structure and its pattern are shown in Fig. 19 and its performance is summarized in Table $\mathrm{V}$.

The structure has a longer length (approximately $62.6 \mathrm{~cm}$ ) compared to the family in Section I and II $(53 \mathrm{~cm})$ therefore it has lower the resonant frequency and efficiency. The reading angle is now around 5 degrees compared to 30 degree in the previous structure.

\section{CONCLUSION}

Various 3D meander line antennas were investigated for possible use in RFID applications. Based on a $3 \times 3 \times 3$ grid pattern for one half of the antenna, all possible combinations were evaluated and those structures with the highest gain, highest impedance and lowest resonant frequency were identified. Moreover, these simulation results are confirmed by practical tests. This allowed an additional design rule to be developed to improve the antenna performance in free space.

The most significant advance has been the design of a semi-planar antenna which functions satisfactorily when placed close to a conductive surface. The antenna has dimensions $2 \times 2 \times 1.2 \mathrm{~cm}^{3}$, resonates at $498 \mathrm{MHz}$, has $62 \%$ efficiency and has a gain of $-3.4 \mathrm{dBi}$ when placed very close to a perfectly conducing surface. The principle of this antenna in free space has been confirmed by monopole practical measurements. It is likely that further optimization can improve the performance of these 3D meander line antennas and a practical test similar to one in [30] can emphasize the results. 


\section{REFERENCES}

[1] G. Marrocco, "RFID antennas for the UHF remote monitoring of human subjects," IEEE Trans. Antennas Propag., vol. 55, pp. 1862-1870, 2007

[2] H. Choo and H. Ling, "Design of electrically small planer antennas using inductively coupled feed," IEE Electron. Lett., vol. 39, no. 22, pp. 1563-1565, Oct. 2003.

[3] K. M. Z. Shams and M. Ali, "Study and design of a capacitively coupled polymeric internal antenna," IEEE Trans. Antennas Propag., vol. 53, no. 3, pp. 985-993, 2005.

[4] K. Fujimoto and J. R. James, Mobile Antenna Systems Handbook. Boston, MA: Artech House, 1994.

[5] K. Fujimoto, A. Henderson, K. Hirasawa, and J. R. James, Small Antennas. New York: Wiley, 1987.

[6] H. R. Chuang and W. T. Chen, "Computer simulation of the humanbody effects on a circular-loop-wire antenna for radio-pager communications at 152, 280 and $400 \mathrm{MHz}$,' IEEE Trans. Veh. Technol., vol. 46, no. 3, pp. 544-559, Aug. 1997.

[7] K. D. Katsibas, C. A. Balanis, P. A. Tirkas, and C. R. Birtcher, "Folded loop antenna for mobile hand-held units," IEEE Trans. Antennas Propag., vol. 46, no. 2, pp. 260-266, Feb. 1998.

[8] J. Rashed and C. T. Tai, "A new class of resonant antennas," IEEE Trans. Antennas Propag., vol. 39, no. 9, pp. 1428-1433, Sep. 1991.

[9] T. Endo, Y. Sunahara, S. Satoh, and T. Katagi, "Resonant frequency and radiation efficiency of meander line antennas," Electron. Commun. Jpn, vol. 83, no. 1, pt. 2, pp. 52-58, Jan. 2000.

[10] G. Marrocco, A. Fonte, and F. Bardati, "Evolutionary design of miniaturized meander-line antennas for RFID applications," in Proc. IEEE AP-S Int. Symp., San. Antonio, TX, Jun. 2002, pp. 362-365.

[11] H. Choo and H. Ling, "Design of planar, electrically small antennas with inductively coupled feed using a generic algorithm," in Proc. IEEE AP-S Int. Symp., Columbus, OH, Jun. 2003, pp. 300-303.

[12] M. Keskilammi and M. Kivikoski, "Using text as a meander line for RFID transponder antennas," IEEE Antennas Wireless Propag., vol. 3, pp. 372-374, 2004.

[13] A. Galehdar, D. V. Thiel, S. G. O'Keefe, and S. P. Kingsley, "Efficiency variation in elelctrically small, meander line RFID antennas," in Proc. IEEE AP-S Int. Symp., Honolulu, HI, Jun. 2007, pp. 2273-2276.

[14] C. T. Rodenbeck, "Planar miniature RFID antennas suitable for integration with batteries," IEEE Trans. Antennas Propag., vol. 54, no. 12, pp. 3700-3706, Dec. 2006.

[15] J. Zhu, A. Hoorfar, and N. Engheta, "Bandwidth, cross-polarization, and feed-point characteristics of matched Hilbert antennas," IEEE Antennas Wireless Propag., vol. 2, pp. 2-5, 2003.

[16] J. Anguera, C. Puente, E. Martinez, and E. Rozan, "The fractal Hilbert monopole: A two-dimensional wire," Microw. Opt. Technol. Lett., vol. 36, pp. 102-104, Jan. 2003.

[17] J. Romeu and S. Blanch, "A three dimensional Hilbert antenna," in Proc. IEEE AP-S Int. Symp., San. Antonio, TX, Jun. 2002, pp. 550-553.

[18] J. M. Gonzalez-Arbesu, S. Blanch, and J. Romeu, "The Hilbert curve as a small self-resonant monopole from a practical point of view," Microw. Opt. Technol. Lett., vol. 39, pp. 45-49, Oct. 2003.

[19] “NEC-Win Plus, User's Manual,” Nittany Scientific, 2003.

[20] E. E. Altshuler, "Electrically small self-resonant wire antenna optimized using a genetic algorithm," IEEE Trans. Antennas Propag., vol. 50, no. 3, pp. 297-300, Mar. 2002.

[21] M. Randall, A. Lewis, A. Galehdar, and D. V. Thiel, "Using ant colony optimization to improve the efficiency of small meander line RFID antenna," in Proc. 3rd IEEE Int. Conf. on e-Sci. and Grid Comput. (e-Sci. 2007), Bangalore, India, Dec. 2007, pp. 10-13.

[22] M. J. Atallah, Algorithms and Theory of Computation Handbook, 1st ed. Boca Raton, FL: CRC Press, 1999.

[23] N. Yonemoto, K. Yamamoto, K. Yamada, and T. Hirata, "RF emission measurement of 433 MHZ RFID Tags for EMI evaluation to onboard instruments of aircrafts," in Proc. 7th Int. Symp. on Electromagn. Compat. and Electromagn. Ecology, Jun. 26-29, 2007, pp. 232-235.

[24] W.-J. Yoon, S.-H. Chung, H.-P. Kim, and S.-J. Lee, Dept. of Comput Eng., Pusan Nat. Univ, "Implementation of a $433 \mathrm{MHz}$ active RFID system for U-Port," in Proc. 9th Int. Conf. on Advanced Commun. Technol., Feb. 12-14, 2007, pp. 106-109.
[25] A. Galehdar, D. V. Thiel, and S. G. O'Keefe, "Antenna efficiency calculations for electrically small, RFID antennas," IEEE Antennas Wireless Propag., vol. 6, pp. 156-159, 2007.

[26] H. A. Wheeler, "Fundamental limitation of small antenna," Proc. IRE, pp. 1479-1484, Dec. 1947.

[27] D. M. Dobkin and S. M. Weigand, "Environmental effects on RFID tag antennas," in IEEE MTT-S Int. Microw. Symp. , Jun. 12-17, 2005.

[28] H.-W. Son and G.-Y. Choi, "Orthogonally proximity-coupled patch antenna for a passive RFID tag on metallic surfaces," Microw. Opt. Technol. Lett., vol. 49, no. 3, pp. 715-717, Mar. 2007.

[29] H. Kwon and B. Lee, "Compact slotted planar inverted-F RFID tag mountable on metallic objects," Electron. Lett., vol. 41, no. 24, pp. 1308-1310, Nov. 2005.

[30] Rao et al., "Antenna design for UHF RFID tags: A review and a practical application," IEEE Trans. Antennas Propag., vol. 53, no. 12, pp. 3870-3876, Dec. 2005.

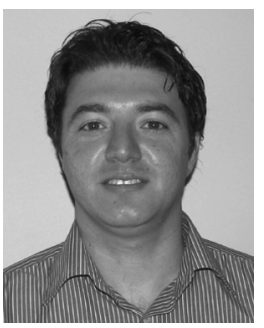

Amir Galehdar was born in Ahvaz, Iran, in 1978. He received the B.Sc. degree in telecommunication engineering from $\mathrm{K}$. N. Toosi University of Technology, Tehran, Iran and the M.Sc. degree in microelectronic engineering from Griffith University, Brisbane, Australia, in 2002 and 2005, respectively. $\mathrm{He}$ is currently working toward the Ph.D. degree at Griffith University.

Since 2004, he has been a member of the Centre for Wireless Monitoring and Applications, Griffith University, where he where is currently working on novel antenna designs for RFID and HF applications. His research interests include antenna development and optimization for RFID UHF passive tags, HF and wireless sensor networks.

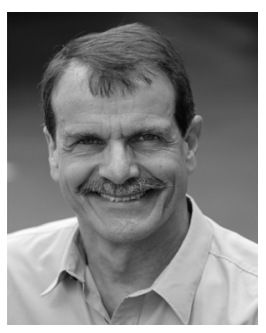

David V. Thiel (M'81-SM'88) graduated in Physics and Applied Mathematics from the University of Adelaide, SA, Australia, and then received the M.S. and Ph.D. degrees from James Cook University, Townsville, Australia.

$\mathrm{He}$ is currently the Director of the Centre for Wireless Monitoring and Applications, Griffith University, Brisbane, Australia. He has published over 80 journal papers, with more than 125 papers presented at international conferences and has co-authored more than eight patent applications. Recently, he was a co-inventor of the new RoHS and WEEE compliant electronics manufacturing technology called "circuits in plastic." His research interests include electromagnetic geophysics, sensor development, electronics systems design and manufacture, and antenna development for wireless sensor networks. He coauthored a book on switched parasitic antennas for cellular communications and is interested in mathematical optimization techniques for antenna design.

Prof. Thiel is a Fellow of the Institution of Engineers, Australia. He is currently the Chair of the IEEE Wave Propagation Standards Committee and serves on the IEEE Antenna Standards Committee.

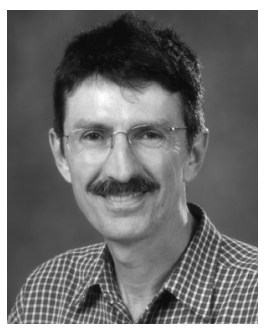

Steven G. O'Keefe (S'85-M'91) was born in Brisbane, Australia. He received the B.Sc. degree in physics, mathematics, and electronics and the Ph.D. degree in geophysical electromagnetic from Griffith University, Brisbane, Australia, in 1985 and 1996, respectively, and the B Sc. degree (Hons.) in electronics and the M.Sc. degree in biomedical electronics from LaTrobe University, Australia, in 1986 and 1990. respectively.

He is currently and Associate Professor and the Deputy Head of the Griffith School of Engineering, Griffith University. His research interests are in the area of compact mobile antenna design and electromagnetic geophysics. 